\title{
A Novel Quasi-Open Loop Architecture for GNSS Carrier Recovery Systems
}

\author{
Muhammad Tahir, ${ }^{1}$ Letizia Lo Presti, ${ }^{1}$ and Maurizio Fantino ${ }^{2}$ \\ ${ }^{1}$ Dipartimento di Elettronica e Telecomunicazioni (DET), Politecnico di Torino, Corso Duca degli Abruzzi 24, 10129 Torino, Italy \\ ${ }^{2}$ Navigation Laboratory, Istituto Superiore Mario Boella, Via P.C. Boggio 61, 10138 Torino, Italy \\ Correspondence should be addressed to Muhammad Tahir, muhammad.tahir@polito.it
}

Received 16 December 2011; Revised 15 March 2012; Accepted 5 April 2012

Academic Editor: Carles Fernández-Prades

Copyright (C) 2012 Muhammad Tahir et al. This is an open access article distributed under the Creative Commons Attribution License, which permits unrestricted use, distribution, and reproduction in any medium, provided the original work is properly cited.

\begin{abstract}
The problem of designing robust systems to track global navigation satellite system (GNSS) signals in harsh environments has gained high attention. The classical closed loop architectures, such as phase locked loops, have been used for many years for tracking, but in challenging applications their design procedure becomes intricate. This paper proposes and demonstrates the use of a quasi-open loop architecture to estimate the time varying carrier frequency of GNSS signals. Simulation results show that this scheme provides an additional degree of freedom to the design of the whole architecture. In particular, this additional degree of freedom eases the design of the loop filter in harsh environments.
\end{abstract}

\section{Introduction}

In global navigation satellite systems (GNSSs) the relative motion of both GNSS satellites and the user causes a Doppler effect, which results in a large frequency shift in the carrier and in the code of the received signal [1]. Precise estimation of this frequency shift is one of the most demanding requirements for GNSS receivers, because only an accurate tracking of the carrier frequency and Doppler shift allows the receiver to work properly, enabling reliable estimates of position velocity and timing (PVT). In any GNSS receiver, the acquisition stage provides an initial coarse estimation of the frequency shift, which is subsequently refined by the tracking systems. They are generally implemented in the form of closed loops, that is, phase lock loops (PLLs) and frequency lock loops (FLL), which track respectively the phase and the frequency of the incoming carrier, [2].

The main building block of a closed loop architecture is the loop filter. The design of a loop filter has been extensively addressed in the literature regarding the continuous-time PLLs, and many results and methods exist for different scenarios. However, modern receivers work in the discretetime domain, and so PLLs and FLLs are digital systems, whose loop filters are often designed starting from some equivalent analog prototypes, by adopting transformation techniques from the analog to the digital domain. These tracking loops are therefore de facto digital approximations of analog loops, whose quality breaks down as the integration time increases. A valid assumption for this approximation is that the product $B T$ between the loop noise bandwidth $B$, and the integration time $T$ remains close to zero. As this product increases, the loop becomes unstable, as discussed in [3]. However, in high dynamic and weak signal applications, it is necessary to work with large $B T$ values. In these cases the design of the loop filters based on analog-to-digital transformations does not work properly. Therefore, other techniques have been proposed in literature, which are more robust when low update rates (long integration times) or large bandwidths are required. They are the controlled root method for the design of digital filters [4], the direct design of loop filters in digital domain by minimization criterion [5], the loop architectures based on Kalman filters [6], and the fuzzy loop architectures [7].

In this paper we propose an alternative solution, based on a novel quasi-open loop architecture, which relieves the stress on the loop filter in terms of stability. In the first step of our study we considered open loop techniques, since some advantages over the closed loop counterpart 
schemes can be achieved. For example, it is a known fact that PLLs are vulnerable to fading effects, typically associated with urban environments, and cycle slips [8]. Moreover, because of their closed loop structure, they need a long acquisition time before attaining the loop lock, and this may be a serious drawback when the Doppler significantly varies within this time interval. The use of open loop architectures can solve these problems. Several open loop architectures have been proposed in literature; most of them are based on the use of the Fast Fourier Transform (FFT) for the estimation of the frequency error between the incoming carrier and a local carrier replica. However, this block processing structure, typical of FFT-based methods, increases the system complexity. In our scheme we do not use FFT for frequency estimation; in our method we estimate the frequency by using conventional PLL/FLL discriminators, for example, the Kay's estimator [9], but with a different update of the frequency of the numerically controlled oscillator (NCO). In particular, instead of updating the NCO at each coherent integration time as in the closed loop architectures, the update is performed after NT integrations intervals, where $N$ is an integer. Between two updating epochs the architecture works as an open loop. Using this approach we are actually working with a three-rate scheme, unlike the conventional two-rate closed loop systems. We have found that this additional degree of freedom can help us to ease the design of the loop filter in weak signal conditions, where it is necessary to extend the coherent integration time.

In a weak signal scenario the extension of the coherent integration time is the only possible option, since the increased processing gain allows the successful recovery of weak and extremely weak signals. However, the maximum coherent integration time in a GNSS receiver is limited by a variety of factors: the presence of navigation data modulation, the stability issues, and the demodulation losses due to a frequency mismatch. This effect is particularly detrimental when the loop update time increases (long integration time) and the incoming carrier has a high Doppler shift [3]. The first problem is usually solved by estimating the navigation data or using external assistance. The main contribution of this paper is related to the second issue, that is, the stability problem, which is solved by adopting an inherently stable architecture. We will show that the proposed architecture can work with extended integration times, which are not possible in the traditional closed loop schemes under the same conditions. In order to solve the third problem, methods of compensation of the user dynamics are necessary. For this purpose, PLLs are generally assisted by other systems, for example, an FLL or a tightly coupled inertial navigation system (INS).

The paper is organized as follows. The GNSS signal model is introduced in Section 2. Then, a general overview of the traditional GNSS carrier recovery systems is presented in Section 3, stressing the aspects which motivate the scheme proposed in this paper. Section 4 shows the novel quasiopen loop scheme for carrier recovery, proposed to overcome some limitations of the traditional schemes. Some simulation results are presented in Section 5, showing the feasibility of the proposed method in terms of tracking jitter performance, in a dynamic scenario and with weak signals. On the basis of these results some possibilities to refine the proposed architecture are also discussed. Conclusions are drawn in Section 6 .

\section{Signal Model}

The main purpose of this paper is to propose a novel architecture for tracking the frequency of the incoming GNSS signal which combines the good properties of both open loop and closed loop architectures. These systems recover the carrier from the received signal, which can be written, after downconversion to intermediate frequency (IF), sampling and quantization, as

$$
x_{w}[n]=\sqrt{2 P} a[n] \cos \left[2 \pi\left(f_{0}+f_{d}\left(n T_{s}\right)\right) n T_{s}+\psi_{s}\right]+w[n],
$$

where $P$ is the total received power of the useful signal, $a[n]=d[n] c[n]$ is the useful signal $(c[n]$ is the code and $d[n]$ the navigation message), $f_{0}$ is an intermediate frequency, $f_{d}\left(n T_{s}\right)$ is a frequency shift (which can be time variant), $\psi_{s}$ is the initial phase (the phase for $n=0$ ), and $w[n]$ is a noise component. This signal is obtained by sampling an analogical signal at a sampling frequency $f_{s}=1 / T_{s}$. The carrier signal is completely characterized by its instantaneous phase

$$
\varphi_{s}\left(n T_{s}\right)=2 \pi\left(f_{0}+f_{d}\left(n T_{s}\right)\right) n T_{s}+\psi_{s} .
$$

The noise term $w[n]$ is a realization of a Gaussian random process $W[n]$, with flat power spectral density $N_{o} / 2$ over the receiver band $B_{r}$, and with power $\sigma_{w}^{2}=N_{o} B_{r} \cdot W[n]$ is not generally a white sequence with a flat power spectral density, due to front-end filtering. However, a white model is justified because the bandwidth of the front-end filter is usually close to the Nyquist sampling frequency, that is, $B_{r}=f_{s} / 2$.

The purpose of the carrier recovery is to estimate $\varphi_{s}\left(n T_{s}\right)$ in order to construct a local oscillator (LO) of the type

$$
x_{\mathrm{cr}}[n]=\cos \hat{\varphi}_{s}\left(n T_{s}\right),
$$

where $\hat{\varphi}_{s}\left(n T_{s}\right)$ is the estimate of $\varphi_{s}\left(n T_{s}\right)$, and the subscript $\mathrm{cr}$ denotes that this signal is recovered from the carrier. Therefore, the LO (producing $x_{\mathrm{cr}}[n]$ ) can be considered the output of the carrier recovery system. We assume that both data and code can be wiped off and we focus on the operations performed by the carrier recovery system by considering the ideal signal

$$
x_{s}[n]=x_{s}\left(n T_{s}\right)=\cos \left[2 \pi\left(f_{0}+f_{d}\left(n T_{s}\right)\right) n T_{s}+\psi_{s}\right],
$$

where $f_{d}\left(n T_{s}\right)$ is an unknown frequency, which varies with an unknown rule, $\psi_{s}$ is also unknown, while $f_{0}$ is a known nominal frequency. In real cases, the signal $x_{s}\left(n T_{s}\right)$ is also affected by noise, and then the carrier has to be recovered from a noisy version of (4).

\section{Traditional Tracking Architectures: Review and Limitations}

The traditional GNSS carrier recovery systems can be classified into two categories: closed loop tracking systems 
and open loop phase and frequency estimators. In this section we briefly review the traditional closed loop systems and the open loop schemes, paying attention to the aspects which motivate the quasi-open loop scheme proposed in the next sections.

3.1. Closed Loop Architectures. Digital phase lock loops (DPLLs) and FLLs lie into the category of the classical closed loop architectures, whose basic scheme is shown in Figure 1. DPLLs are able to track both the instantaneous phase and frequency of the incoming carrier, while FLLs are only able to track the carrier frequency.

In the following we briefly describe the DPLL operations (the operations performed by FLLs are almost similar except for the discriminator). The estimation of the instantaneous phase is generally performed by a DPLL, which consists of a number of subsystems: (a) the phase estimator/discriminator, (b) the loop filters $F(z)$, (c) the local oscillator (LO) also called numerically controlled oscillator (NCO). The input signal $x_{w}[n]$ is first multiplied by the local carrier $x_{L}[n]$ generated by the NCO, and integrated by the integrate and dump block. During this integration process, $L$ input samples are processed and used to produce the prompt correlator output $y_{\phi}[k]$. The corresponding integration time is $T=L T_{s}$. At this point the sampling rate of the system changes from $1 / T_{s}$ (time domain $n$ ) to $1 /\left(L T_{s}\right)$ (time domain $k)$. The instantaneous phase is estimated from $y_{\phi}[k]$ by the phase discriminator. This estimate is filtered through the loop filter $F(z)$, with a loop noise bandwidth $B$, and the filter output is used to drive the $\mathrm{LO}$ for the carrier generation. This estimate is then progressively updated using the information provided by the new correlator output in a closed loop manner. Below we present two key points that motivate the use of a quasi-open loop scheme (for GNSS carrier recovery systems) rather than a closed loop one.

It is important to stress that, in the traditional closed loop carrier recovery systems, the LO role is twofold: in fact it is both the output of the system and an integral part of the phase estimator. In the quasi-open loop structure proposed in this paper these two roles will be decoupled, as explained in Section 4. This is the first key point of our method, as will be clear in the next sections.

The loop filter $F(z)$ is the most critical block, whose function is also twofold. Firstly, as the received signal and, thus, the discriminator output is corrupted by thermal noise, the filter is required to provide a degree of noise rejection. Secondly, it enables the processing of higher order dynamics. Transformation methods from the Laplace domain to the $Z$-domain are widely used to design loop filters [2]. These methods simply provide a discrete version of the loop filters that have been previously designed for the analog loops. However, this approach neglects both the inherent delay in the digital loop and the variation in the open loop gain due to the NCO update interval. In [3] it has been shown that the transformation methods properly work only if $B T$ is close to zero, where $B$ is the loop noise bandwidth and $T$ is the integration time. As $B T$ increases, the effective loop noise bandwidth and the closed loop pole locations deviate from the desired ones and eventually the loop becomes unstable, as explained in [3]. The maximum achievable $B T$ value depends on the type of the Laplace- $Z$ transformation and on the characteristics of the original continuous-time filter. For most communication applications, this condition is satisfied because $B T$ remains close to zero. Instead, for some new GNSS applications, such as for weak signal tracking and extremely high dynamic applications, larger $B T$ values are required.

Simulation experiments have been carried out to analyze the behavior of an FLL for increasing values of $B T$. The incoming GNSS signal has been generated with a carrier to noise ratio $C / N_{o}=40 \mathrm{~dB}-\mathrm{Hz}$ and with a ramp-type time varying frequency with a slope equal to $10 \mathrm{~Hz} / \mathrm{s}$. In order to track this frequency evolution, we have used a 2nd order FLL with a loop noise bandwidth $B=10 \mathrm{~Hz}$, and we have changed $B T$ (by modifying the integration time) to put progressively more stress on the loop. The frequency estimated by the FLL is shown in Figure 2 for three different values of $B T$, together with an estimate of $C / N_{o}$. The latter can be used as an indicator to check if the system is in lock state or not. These results show that the loop loses the lock and the FLL is no longer able to track the input frequency when $B T=0.3$, as indicated by the estimated $C / N_{o}$. It is important to emphasize that the loop filter is not the only module in the closed loop scheme responsible for the loss of lock and the stability problems. The loop can also lose lock or can become unstable because of other factors, but here we concentrate on the effect of the loop filter design based on transformation methods on loop. To solve this problem, some techniques for designing the loop filters have been proposed in the literature, for example, the controlled root method, the direct design of loop filters in digital domain based on a minimization criterion, the loop architectures based on Kalman filters, and the fuzzy loop architectures. An alternative approach is to use the scheme proposed in Section 4, which simplifies the design of the loop filter. This is the second key point of our method.

3.2. Open Loop Architectures. In order to estimate the time delay and the carrier frequency from the incoming signal, the open loop schemes usually operate on batches of the incoming signal, as depicted in Figure 3. The open loop approach does not separate acquisition and tracking stages, as explained in [10]. An input signal batch is correlated with batches of a signal replica in order to obtain an entire 3-D image of the signal, whose dimensions are the code shift, the Doppler shift, and the signal energy. This batch-based correlation uses joint time-frequency domain techniques to allow some forms of parallel computing based on FFT. After the 3-D image has been obtained, batch estimators are applied to the 3-D function to compute the signal parameters. They operate by searching for the location of the maximum energy of the $3-\mathrm{D}$ function and provide an estimate of the parameters $\Delta \psi, \Delta f[k]$, and the code delay corresponding to this location. Since these methods mainly rely on FFT-based correlators for the 3-D image computation, they usually exhibit a large computational complexity, which enormously increases as the batch size 


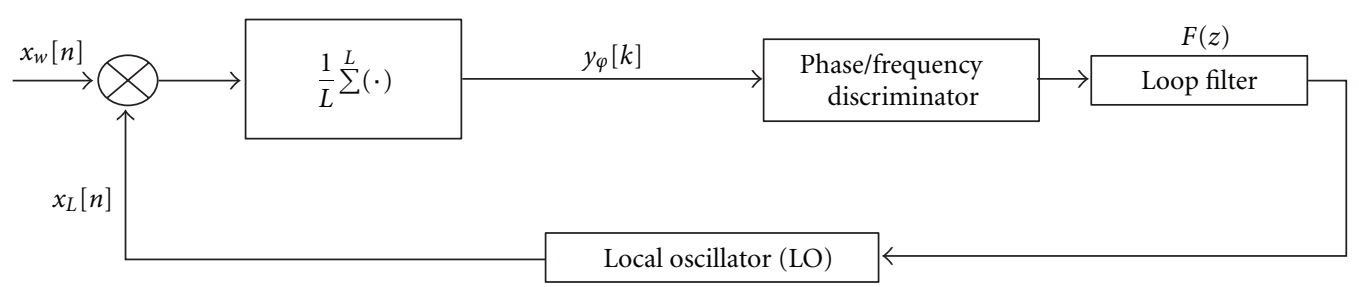

FIgURE 1: Basic classical closed loop carrier tracking architecture.

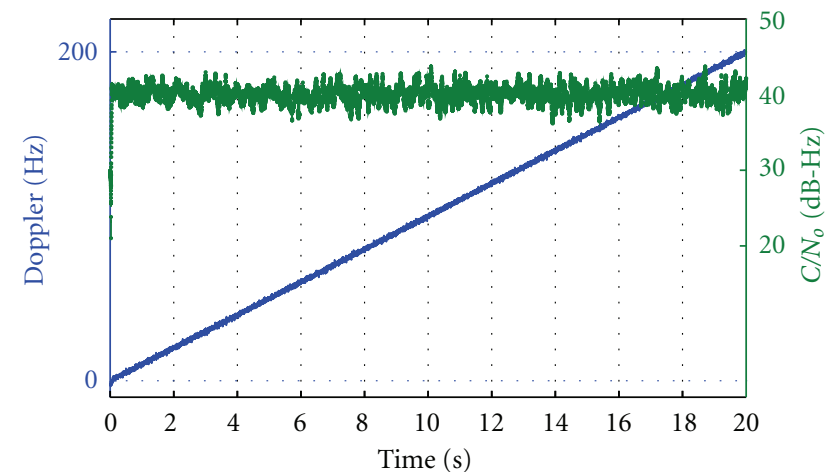

(a) $B T=0.01$

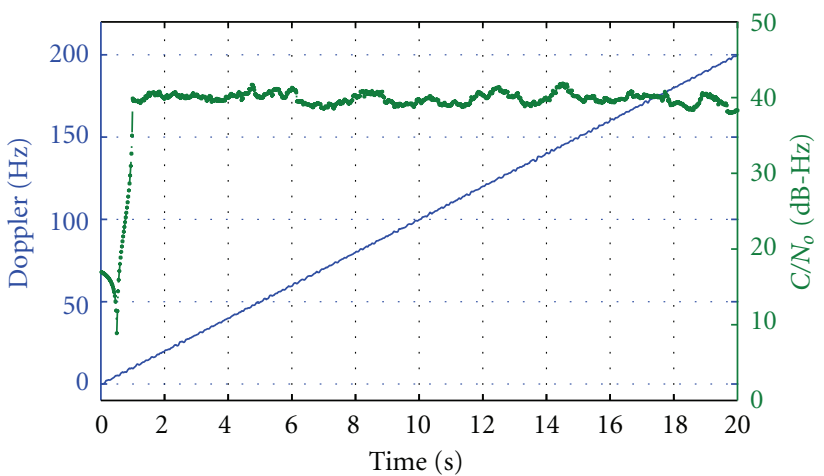

(b) $B T=0.2$

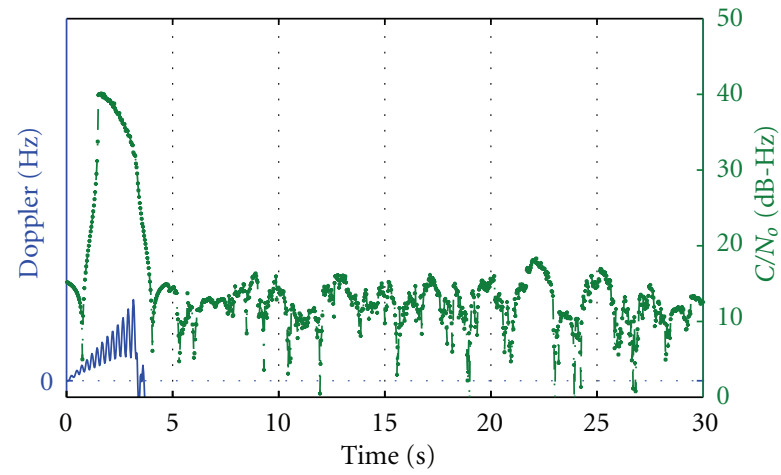

(c) $B T=0.3$

FIGURE 2: 2nd order FLL tracking tesults for input ramp frequency of $10 \mathrm{~Hz} / \mathrm{s}, C / \mathrm{N}_{o}=40 \mathrm{~dB}-\mathrm{Hz}, B=10 \mathrm{~Hz}$ and different integration Times.

increases. This is the main reason to avoid the use of these open loop processing techniques in real time receivers.

\section{Proposed Quasi-Open Loop Architecture}

In this section we propose a quasi-open loop scheme for carrier recovery in a GNSS receiver. We start our discussion from a basic open loop scheme, which utilizes a conventional discriminator. Then, after analyzing the behavior of the discriminator for the frequency estimation, we propose a quasi-open loop scheme for the continuous tracking of the incoming carrier frequency.

\subsection{Basic Open Loop Scheme Utilizing Conventional Discrim-} inators. The main idea of an open loop carrier recovery scheme that utilizes a conventional discriminator is depicted in Figure 4.
The main blocks of this scheme are the following.

(i) The local oscillator which generates the local signal $x_{L}[n]$. This complex LO is denoted by $\mathrm{LO}(\mathrm{E})$, to emphasize its role in the process of phase estimation.

(ii) The mixer which performs the multiplication between the local oscillator and the incoming signal.

(iii) The accumulator which accumulates $L$ values of the signal $y[n]$; this block is often called integrator as it is equivalent to an integral in the continuous time domain. The integrator output is provided at the epochs $n=L, 2 L, \ldots, k L, \ldots$.

(iv) The discriminator, which provides an estimate of the instantaneous phase/frequency of the incoming signal. It receives an input (the integrator output) at a rate which depends on the value of $L$.

(v) The open loop smoothing filter. 


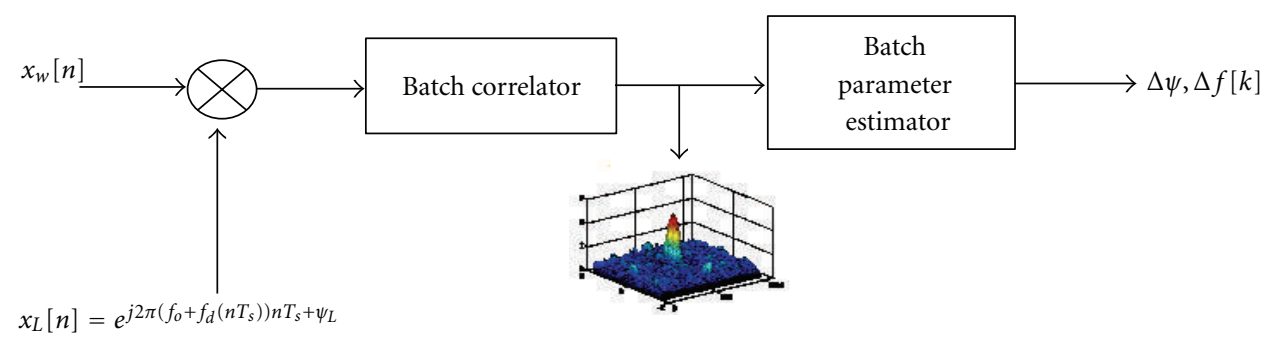

FIGURE 3: a generic open loop GNSS receiver.

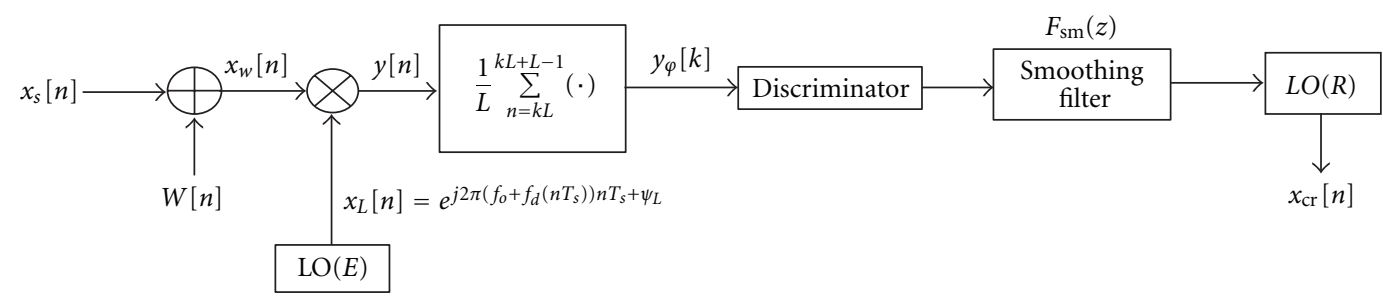

FIgURE 4: A generic open loop carrier recovery scheme utilizing conventional discriminators.

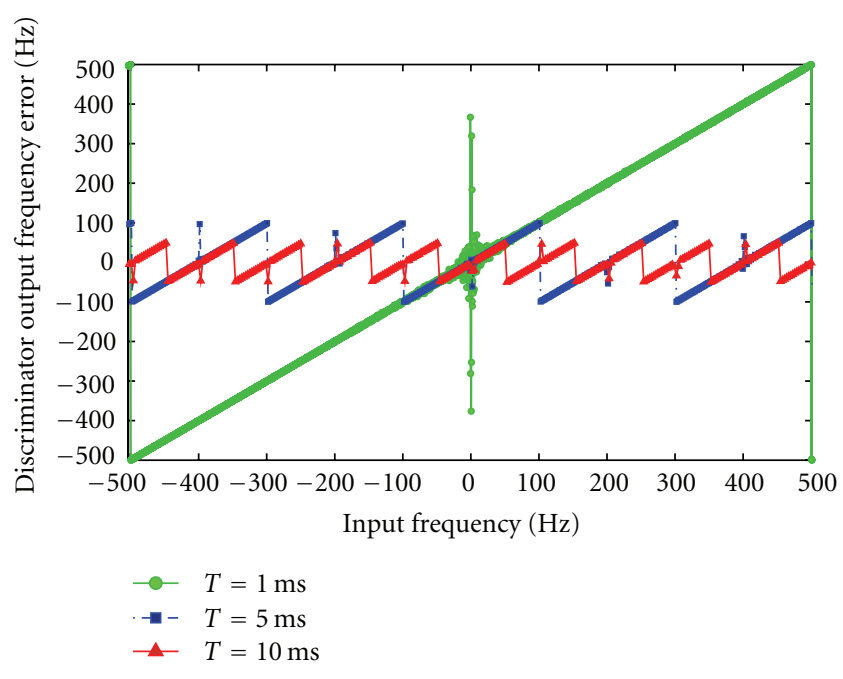

FIGURE 5: Frequency discriminator for different integration times.

(vi) The local oscillator $\mathrm{LO}(\mathrm{R})$, which provides a local carrier $x_{\mathrm{cr}}[n]$, recovered from the incoming signal.

This scheme differs from its closed loop counterpart since we use each new phase/frequency estimate to update a separate local oscillator (the $\mathrm{LO}(\mathrm{R})$ block), instead of feeding back the local oscillator $\mathrm{LO}(\mathrm{E})$ that is embedded in the estimation module. This system works at two different rates, that is, in two different discrete-time domains: the mixer and the integrator works in the $n$ domain with a rate $r_{n}$, while the discriminator works in the $k$ domain, with a rate $r_{k}=$ $r_{n} / L$. Once the discriminator has estimated the instantaneous phase/frequency of the incoming signal, the carrier can be recovered by building a sinusoidal signal, $x_{\mathrm{cr}}[n]$. This is the recovered LO, denoted as $\mathrm{LO}(\mathrm{R})$, whose update rate is $r_{k}$. In a
DPLL, the $\mathrm{LO}(\mathrm{R})$ is also used to update the signal $x_{L}[n]$, then $\mathrm{LO}(\mathrm{E})$ and $\mathrm{LO}(\mathrm{R})$ coincide.

In our scheme we decouple $\mathrm{LO}(\mathrm{R})$ and $\mathrm{LO}(\mathrm{E})$, so as to design independently their denoising filters and update rates. This idea derives from the consideration that the update rate of $\mathrm{LO}(\mathrm{R})$ depends on the input dynamic, while the update rate of $\mathrm{LO}(\mathrm{E})$ depends on the frequency range the discriminator is able to process. It is evident that the two update rates are related, but the requirements can be different. Similar considerations can be done for the denoising filters.

Below we perform an analysis of the integrator and discriminator outputs which will help us to highlight the main factors that are needed to recover a continuous carrier. The analysis is performed in the ideal case of no noise. In this case, the integrator outputs of Figure 4 can be written in complex form as

$$
y_{\varphi}[k]=\frac{1}{2 L} \sum_{n=k L}^{(k+1) L-1} e^{j\left(2 \pi \Delta f\left(n T_{s}\right) n T_{s}+\Delta \psi\right)},
$$

where

$$
\begin{gathered}
\Delta f\left(n T_{s}\right)=f_{L}\left(n T_{s}\right)-f_{d}\left(n T_{s}\right), \\
\Delta \psi=\psi_{L}-\psi_{s} .
\end{gathered}
$$

In theory (5), should contain also a double frequency component, which, however, can be neglected as it is filtered out by the integrator. If $\Delta f\left(n T_{s}\right)$ is a constant $\Delta f$, the summation in (5) can be written as a geometrical progression, from which a closed-form expression can be found. In fact by writing $i=n-k L$, (5) becomes

$$
\begin{aligned}
y_{\varphi}[k] & =\frac{1}{2 L} \sum_{i=0}^{L-1} e^{j\left(2 \pi(i+k L) \Delta f T_{s}+\Delta \psi\right)} \\
& =e^{j\left(2 \pi \Delta f k L T_{s}+\Delta \psi\right)} e^{j \pi \Delta f T_{s}(L-1)} \frac{\sin \pi \Delta f L T_{s}}{2 L \sin \pi \Delta f T_{s}}
\end{aligned}
$$




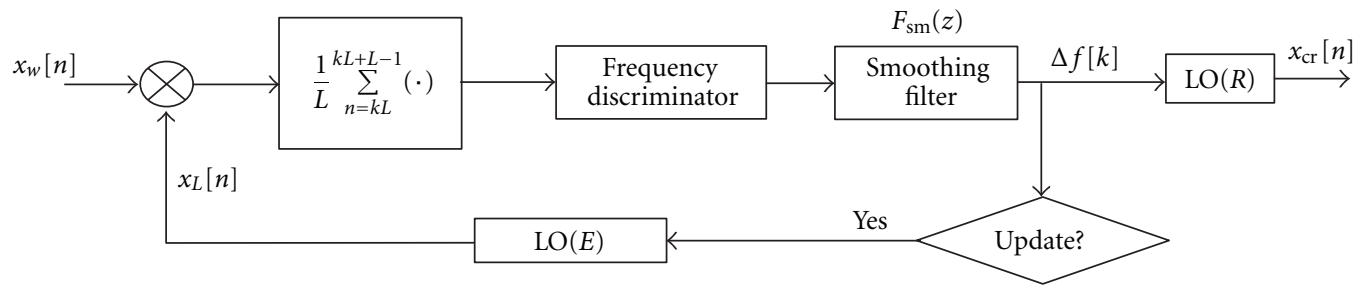

Figure 6: A quasi-open loop frequency estimator.

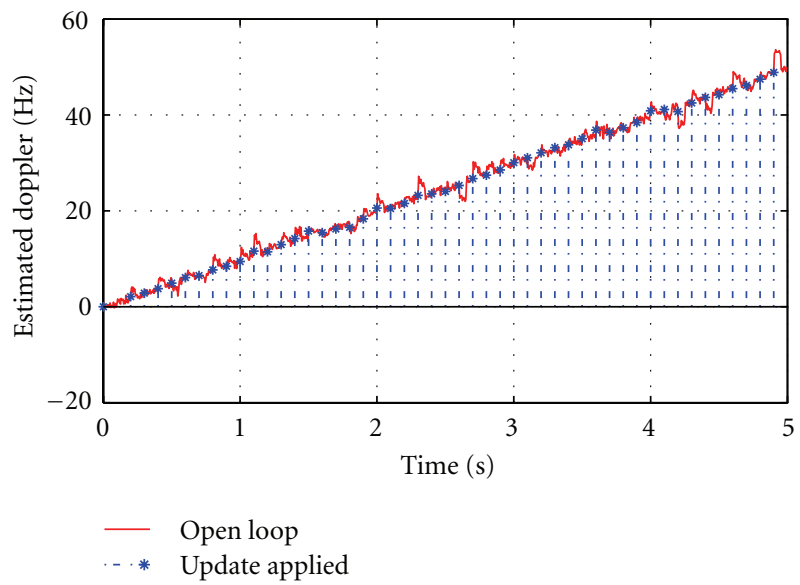

(a)

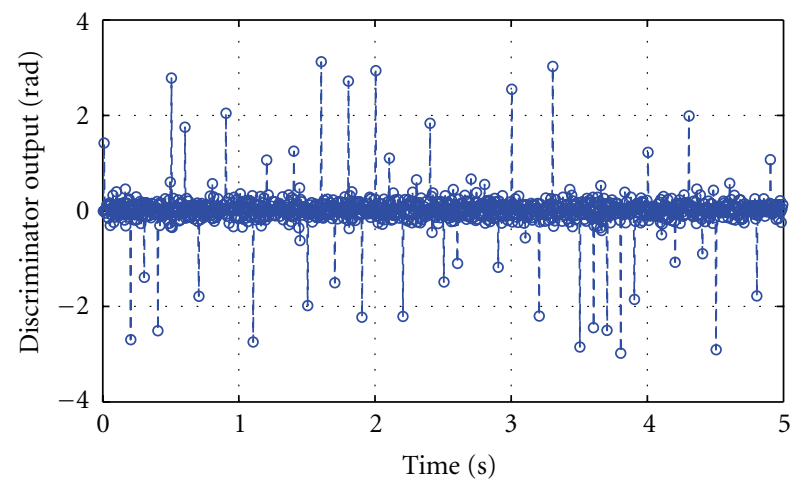

(c)

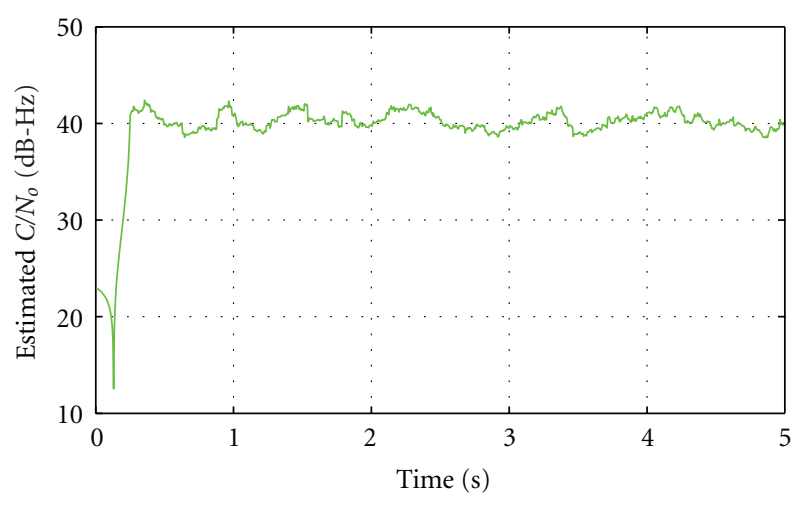

(b)

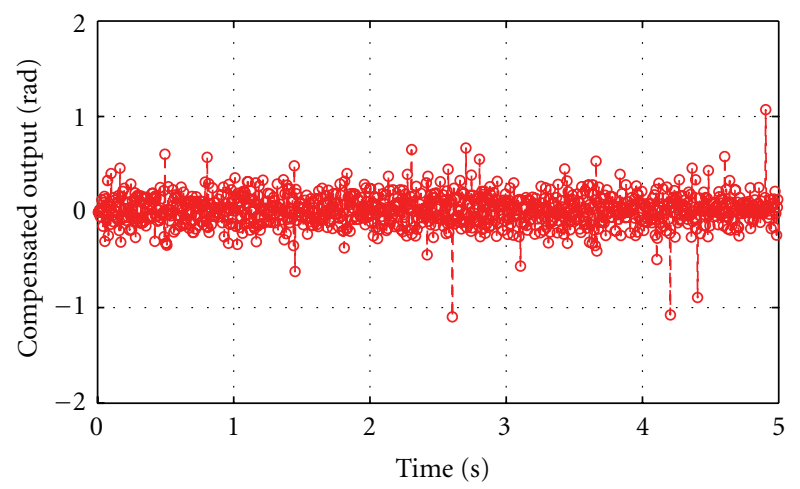

(d)

Figure 7: Proposed scheme tracking an input frequency ramp of $10 \mathrm{~Hz} / \mathrm{s}, \mathrm{C} / \mathrm{N}_{o}=40 \mathrm{~dB}-\mathrm{Hz}, T=5 \mathrm{~ms}$.

whose instantaneous phase is

$$
\varphi_{\text {inst }}[k]=2 \pi \Delta f\left(k L+\frac{L-1}{2}\right) T_{s}+\Delta \psi .
$$

If $\Delta f\left(n T_{s}\right)$ is approximately constant in the integration interval $L T_{s}$, that is $\Delta f\left(n T_{s}\right) \cong \Delta f[k]$ for $k L \leq n \leq(k+$ 1) $L-1$, then it is possible to write

$$
\varphi_{\text {inst }}[k] \cong 2 \pi \Delta f[k]\left(k L+\frac{L-1}{2}\right) T_{s}+\Delta \psi .
$$

This phase contains an integer number $L_{k}$ of cycles plus a fractional part, from which

$$
\varphi_{\text {inst }}[k]=L_{k} 2 \pi+\Phi\left(n T_{s}\right),
$$

where

$$
\Phi\left(n T_{s}\right)=\bmod \left(\varphi_{\text {inst }}[k], 2 \pi\right)
$$

In the classical DPLL schemes for GNSS applications, this phase is estimated at the time epochs $n=k L$, for $k=0,1, \ldots$, as

$$
\Phi\left(k L T_{s}\right)=\arctan \frac{Y_{Q}[k]}{Y_{I}[k]},
$$

where $Y_{I}[k]=\mathcal{R}\left(y_{\varphi}[k]\right)$ and $Y_{Q}[k]=\ell\left(y_{\varphi}[k]\right)$. This discriminator function can also be substituted by other operations which approximate the arctan function. At each 


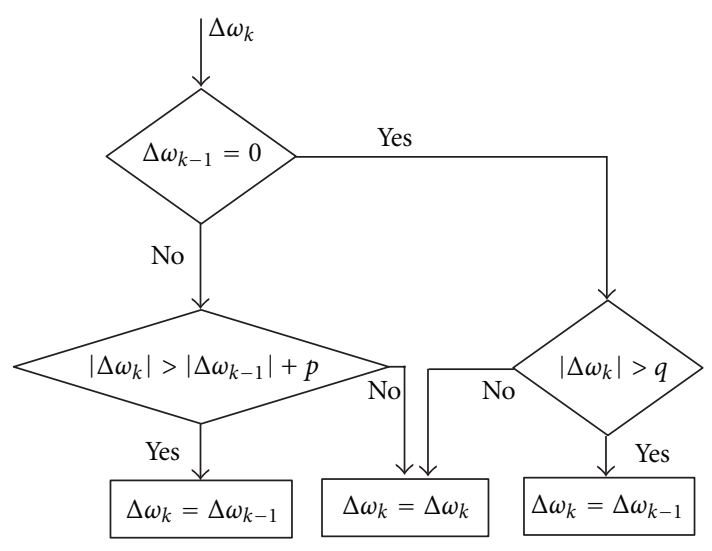

Figure 8: A simple algorithm to detect and remove discriminator output outliers.

epoch $k$, (12) provides a phase value available at the discretetime instant $(k+1) L$ and representative of the instantaneous residual phase at the time instant

$$
l_{k} T_{s}=T_{s}\left[(k+1) L-\frac{(L+1)}{2}\right]
$$

that is, the residual phase information is provided with a delay of $(L+1) / 2$. Notice that the recovered carrier $x_{c r}[n]$ has to be generated at each time instants $t_{n}=n T_{s}$, as indicated in (3). This means that the missing values of the instantaneous phase have to be evaluated in some way. This problem can be easily solved if the unknown frequency $\Delta f\left(n T_{s}\right)$ can be estimated from the outputs of the discriminator function (12), used for the instantaneous phase estimation. This part is described below.

In order to estimate the instantaneous frequency, a quantity $y_{\varphi}[k] y_{\varphi}^{*}[k-1]$ is at first evaluated, and then its phase is extracted using an arctan phase discriminator. This is the usual operation performed by a 4-quadrant arctan discriminator of the type, [2],

$$
\Delta f[k]=\frac{1}{2 \pi T} \arctan \left(\frac{\ell\left(y_{\varphi}[k] y_{\varphi}^{*}[k-1]\right)}{\mathcal{R}\left(y_{\varphi}[k] y_{\varphi}^{*}[k-1]\right)}\right) .
$$

Although this discriminator is termed as maximum likelihood estimator by [2], it is important to mention that this estimator only achieves the Cramer-Rao lower bound at a sufficiently high $C / N_{o}$, as we have demonstrated in [11]. Notice that (14) provides the frequency value without evaluating the instantaneous phase, just applying the integrator output to (14). Another possibility for the frequency evaluation is to operate on the output of the phase discriminator given in (12). However, since in a frequency discriminator only the phase difference is of interest, the discriminator given in (14) is generally more convenient, as it inherently reduces the phase wrapping problem [12]. Once the estimate of $\Delta f[k]$ is available, the carrier can be continuously recovered, at each instant, by the oscillator $\mathrm{LO}(\mathrm{R})$.
Before addressing the role of the filter in this scheme we want to highlight some limitations encountered when a carrier with a time varying Doppler frequency has to be tracked. The main problem with this type of scheme is that we cannot use it alone or without a closed loop updating because of the limited linear region of the discriminator function. In fact, as the difference $\Delta f[k]$ between the frequencies of the incoming and local carriers increases with time, the system tends to operate outside the linear region, where tracking is no longer possible. Moreover, this linear region reduces as the coherent integration time $T$ increases. Therefore, it is not possible to track the frequency evolution without updating the frequency of the local oscillator $\mathrm{LO}(\mathrm{E})$. All the conventional discriminators exhibit the same behavior, as discussed in [2].

As an example, Figure 5 shows the output of the discriminator, given by (14), for different integration times. This behavior is observed when we try to use the scheme, given in Figure 4, for tracking a time varying input Doppler at $C / N_{o}=50 \mathrm{~dB}-\mathrm{Hz}$.

4.2. Modified Scheme: Quasi Open Loop Scheme. A possible method to overcome the problem of tracking a time varying frequency is to update the frequency of the local oscillator LO(E) after $N$ epochs, instead of updating it at each epoch, as in the closed loop systems. This means that we are proposing a scheme working with three different rates, as opposed to the typical closed loop schemes working with two different rates. In the proposed scheme, shown in Figure 6, the additional update rate of the $\mathrm{LO}(\mathrm{E})$ frequency, equal to $r_{u}=r_{k} / N$, is introduced.

The real motivation behind this three rate schemes is to make the integration time $T$ and the NCO update interval independent of each other, obtaining in turn an additional degree of freedom. The latter allows us to ease the design of the loop, as it will be shown below. The system can be considered quasi-open, as it works as an open loop between two updating epochs.

Notice that in our scheme only the frequency of the NCO is updated, while the phase is kept continuous at each epoch, regardless of whether the updating is or is not applied. The value of $N$ depends on the specific application. For example, in case of high dynamics we need to update the $\mathrm{NCO}$ frequency more frequently, so a smaller value of $N$ is necessary. Furthermore, we can also decide to have either regular update intervals, that is, to update after each $N$ epoch, or irregular intervals which depend on the incoming Doppler evolution. In this study we have only considered regular update intervals.

4.3. Choice and Design of Loop Filter. The scheme of Figure 6 allows us to approach the design of the loop filter, taking into account that now its role is only to reduce the effect of noise on the frequency estimate. This is possible thanks to the available additional degree of freedom of the quasi-open scheme. The filter can be considered as a part of the discriminator, whose only task is to smooth the discriminator output. To this purpose, any reasonable choice 

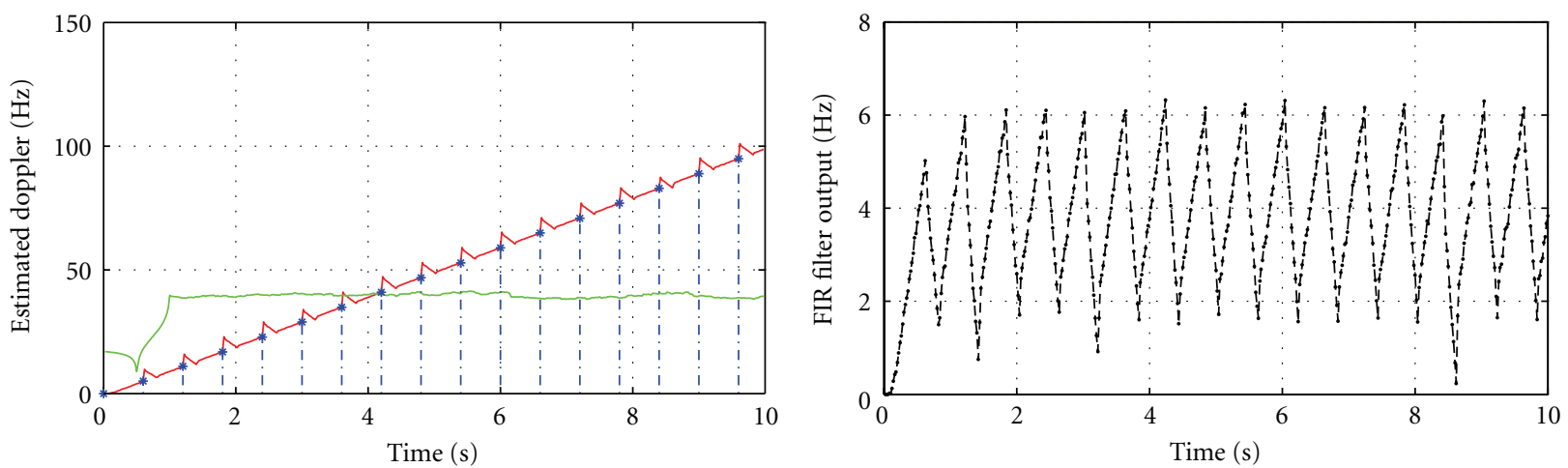

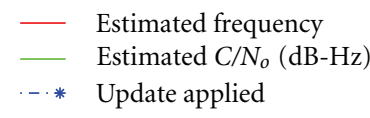

(a)

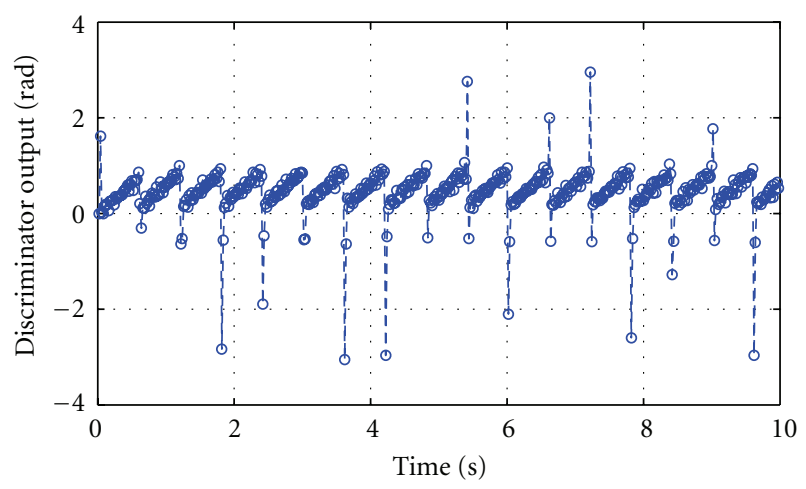

(c)

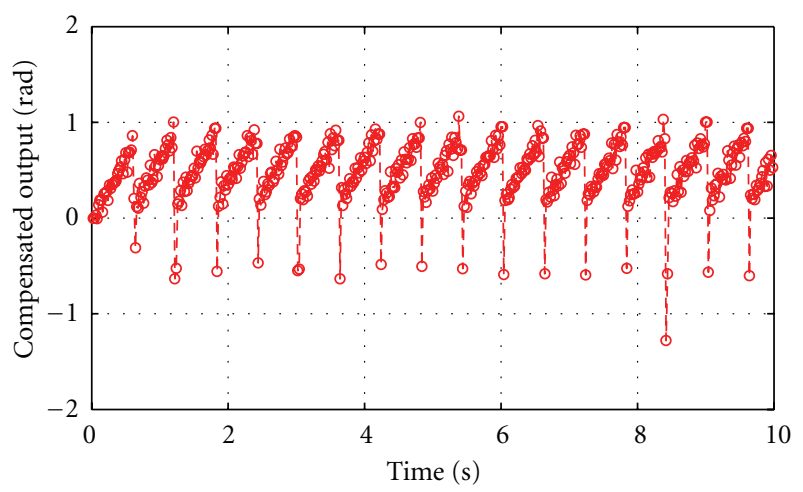

(d)

Figure 9: Proposed Scheme tracking an input frequency ramp of $10 \mathrm{~Hz} / \mathrm{s}, \mathrm{C} / \mathrm{N}_{o}=40 \mathrm{~dB}-\mathrm{Hz}, \mathrm{T}=20 \mathrm{~ms}$.

of the filter structure and parameters can be adopted, taking into account the specific application. Since finite impulse response (FIR) filters are simple to design, always stable, relatively insensitive to quantization and can have linear phase, we decided to explore the possibility to use them for denoising.

The transfer function $F_{\mathrm{sm}}(z)$ of an FIR filter is

$$
F_{\mathrm{sm}}(z)=\sum_{i=0}^{L_{\mathrm{sm}}-1} b_{i} z^{-i}
$$

where $L_{\mathrm{sm}}$ denotes the number of taps and $b_{i}$ are the filter coefficients, which become $b_{i}=1 / L_{\mathrm{sm}}$ in the simplest case of a moving average (MA) filter. An MA-FIR filter is an excellent smoothing filter, but its frequency roll-off is slow and its stopband attenuation is ghastly, making it a scarcely effective low-pass filter. This is a typical result, as a digital filter can be generally optimized for time or frequency domain performance, but not for both. Since in our application the filter task is to mitigate the noise effect, the choice of an MA-FIR structure is justified. Notice that the purpose of this paper is to show the feasibility of a quasi-open architecture, then we did not concentrate on the optimization of the structure of the smoothing filter, which could be also implemented with infinite impulse response (IIR) filters or with more optimized versions of FIR filters.

A possible consideration for choosing the type of filter could be to keep the computational complexity as low as possible. This complexity can be attributed to the number of operations (additions and multiplications) needed to compute the filter response. For digital filters, the computational complexity is more or less proportional to the number of filter coefficients. Usually, we need more than 5 taps to get good smoothing results in case of MA-FIR filters. With these values the computational complexity of a quasi-open loop scheme, which utilizes a smoothing MA-FIR filter, will be slightly higher than the one of an FLL utilizing 2nd order IIR filters. But, as mentioned above, we have many other advantages of using FIR filters as compared to IIR filters.

It is important to mention that the use of these filters changes the philosophy of the loop filter. As explained earlier, in a closed loop architecture the filter has a twofold task: one is to reduce the effect of the noise and the second one is to control the loop dynamics. In the schemes with a smoothing MA-FIR filter the loop dynamics is solely determined by the frequency discriminator. For example, it has been shown that the estimator proposed in (14) is unbiased if we have to track a constant frequency shift or a linear frequency ramp. But its 


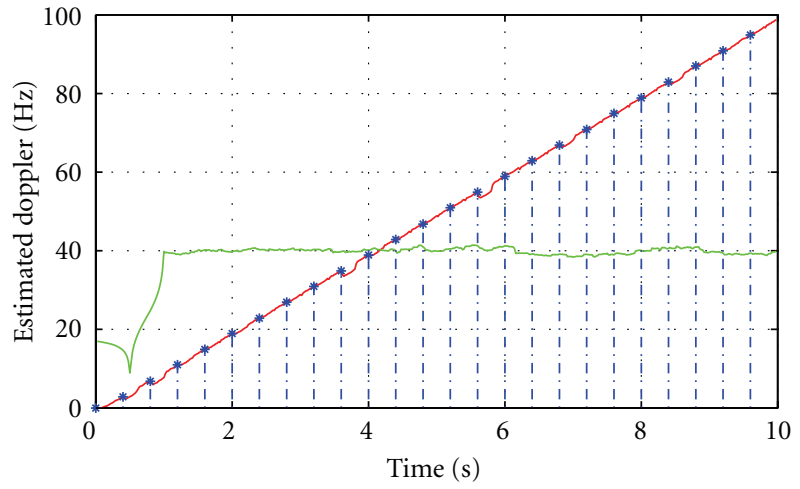

$\begin{array}{ll}- & \text { Estimated frequency } \\ - & \text { Estimated } \mathrm{C} / \mathrm{N}_{o}(\mathrm{~dB}-\mathrm{Hz}) \\ -\cdot \cdot * & \text { Update applied }\end{array}$

(a)

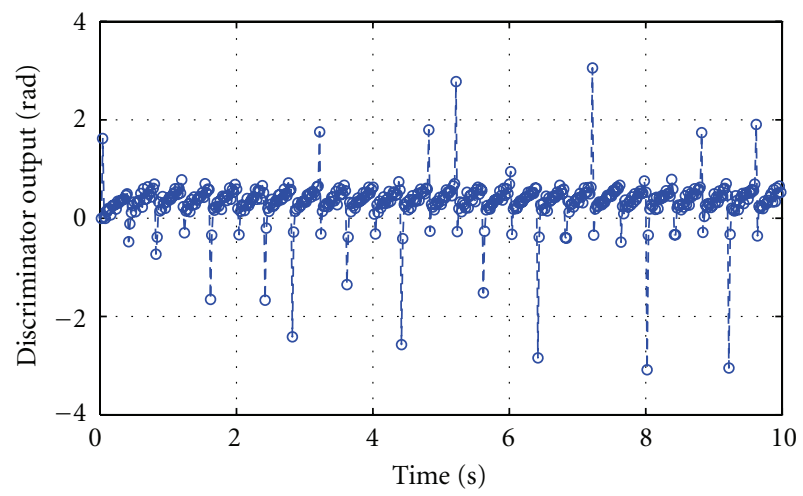

(c)

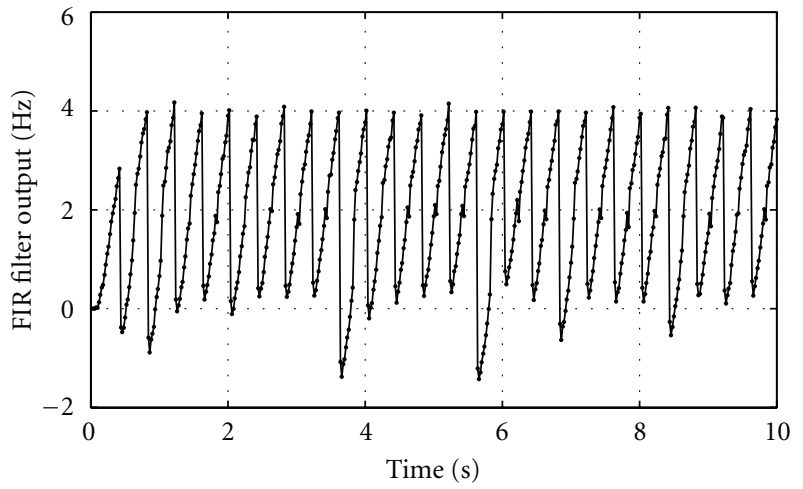

(b)

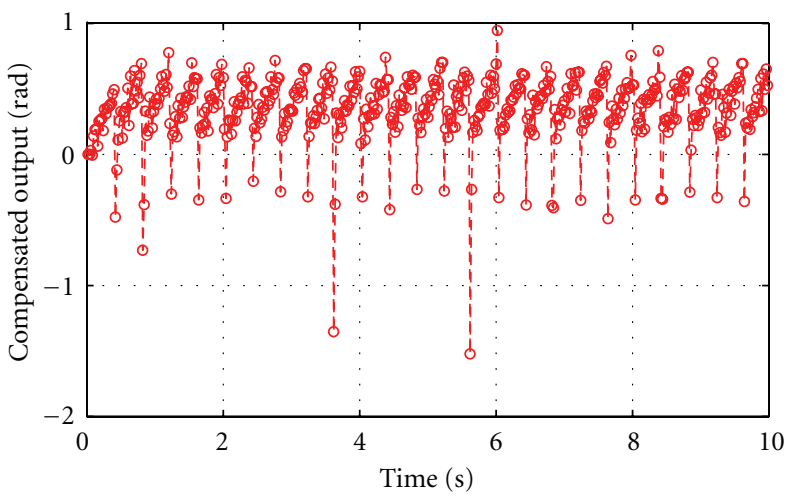

(d)

Figure 10: Proposed scheme tracking an input frequency ramp of $10 \mathrm{~Hz} / \mathrm{s}, C / N_{o}=40 \mathrm{~dB}-\mathrm{Hz}, T=20 \mathrm{~ms}$.

estimate is biased when we have to track an input frequency with a quadratic component [8]. This is not a problem in our scheme as we can accommodate a higher order dynamics by taking additional measures. For example, we can either change the design of the discriminator, as discussed in [13], or we can insert a new block after the FIR filter to account for the higher dynamics. This problem has not been considered in this study, which is mainly devoted to the feasibility and performance of the quasi-open structure.

\section{Results and Discussions}

In this section we present some simulation results obtained by using this new type of quasi-open loop frequency estimator. We start from a basic scheme using an MA-FIR filter, we observe the results, and we introduce some modifications in the basic scheme to improve its performance. The system performance is described in terms of tracking jitter, also taking into account weak signal scenarios.

5.1. Some Implementation Aspects. In the first simulation example, a GNSS-like signal was generated with a ramptype time-varying Doppler shift, with a slope of $10 \mathrm{~Hz} / \mathrm{s}$.
The signal was processed by a quasi-open loop frequency estimator, followed by a smoothing MA-FIR filter with $L_{\mathrm{sm}}$ $=10$ taps. In the first stage (after switch-on) the system is completely open, and, after a time interval equal to the transient of the FIR filter, starts updating the loop. The updating is repeated at each integration interval NT.

The results with $N=20$ are shown in Figure 7, which shows that the proposed scheme is able to successfully track the input Doppler frequency $7(\mathrm{a})$.

However, observing the results of Figure 7, we recognize that some problems are associated with this type of scheme. First of all, at each new update there is a jump in the discriminator output 7 (c). These jumps give rise to undesired outliers which depend on the type of updating. These outliers can be compensated using the scheme shown in Figure 8, which adopts a simple strategy to eliminate any kind of anomaly, based on the comparison between the current and the previous discriminator outputs, indicated in Figure 8, respectively, as $\Delta \omega_{k}$ and $\Delta \omega_{k-1}$, and measured in rad units. The sudden jumps are eliminated by setting two thresholds $p$ and $q$, whose values depend on the rate of the input Doppler frequency. We have set both of them equal to $\pi$ in all the results presented in this paper. The 


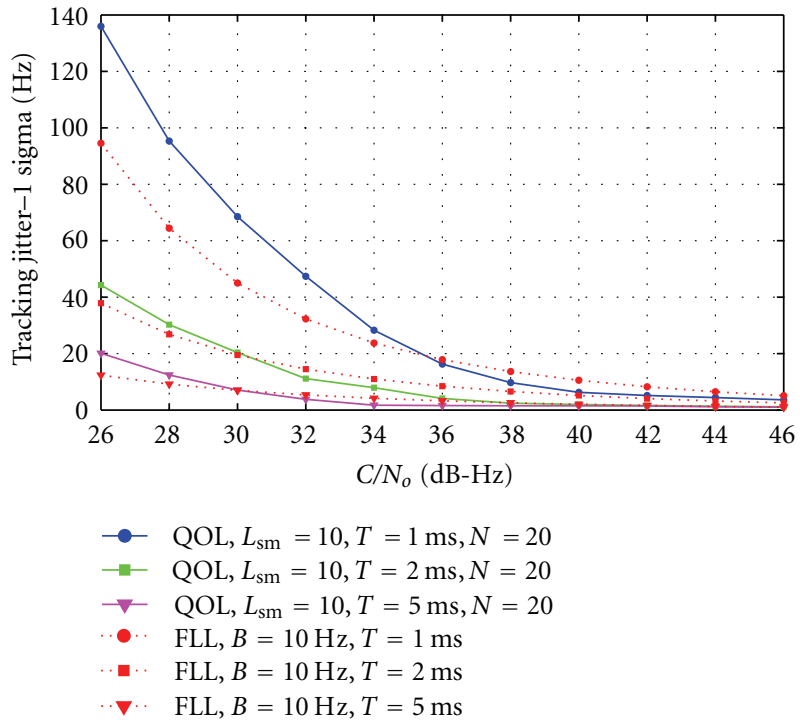

FIGURE 11: Tracking jitter performance.

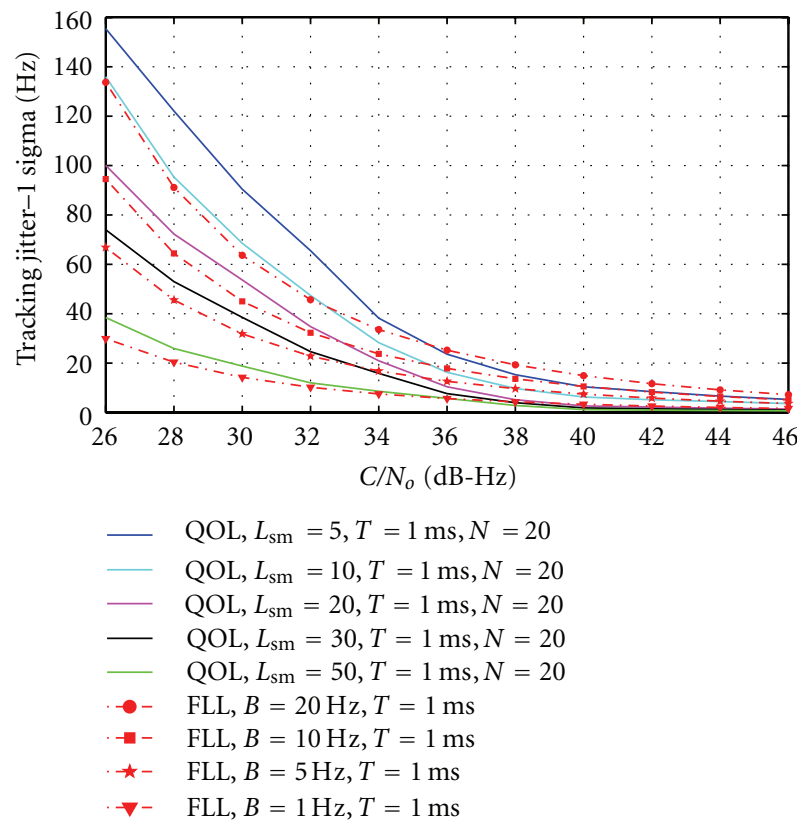

FIGURE 12: Tracking jitter performance with same integration time.

compensated discriminator output shown in Figure 7(d) proves the effectiveness of the method.

The second problem associated to this type of scheme is related to the inherent time-transient of any FIR filter, which generates some regular slowly decreasing jumps at each new updating interval. This is due to the fact that the filter output experiences a transient, which vanishes only when the filter memory (equal to the filter length) is completely filled with input samples. This effect is clearly visible in Figure 9(a) where the integration time is $T=20 \mathrm{~ms}$. A possible solution to mitigate this problem is to use a moving average FIR filter

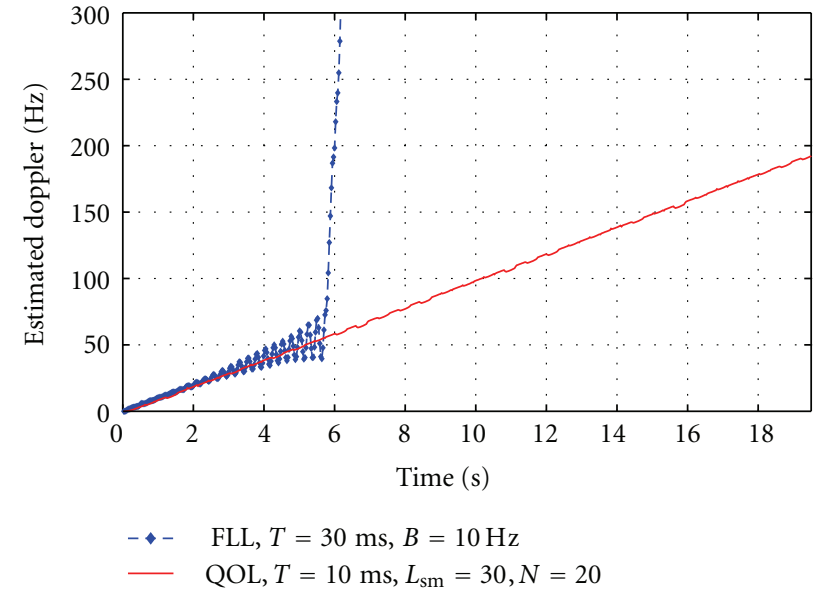

FIGURE 13: FLL versus QOL tracking results for incoming Doppler variation of $10 \mathrm{~Hz} / \mathrm{s}, \mathrm{C} / \mathrm{N}_{o}=40 \mathrm{~dB}-\mathrm{Hz}$.

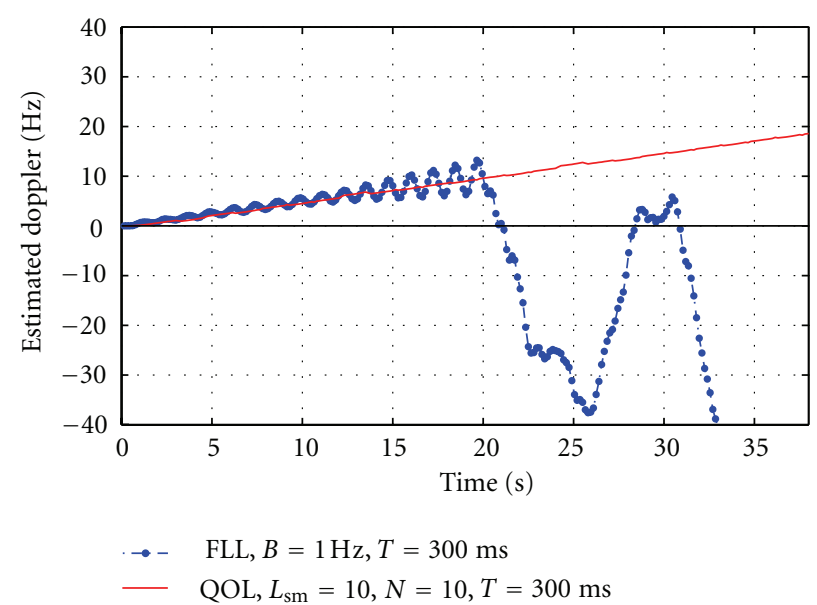

FIGURE 14: FLL versus QOL tracking results for incoming Doppler variation of $0.5 \mathrm{~Hz} / \mathrm{s}, \mathrm{C} / \mathrm{N}_{o}=30 \mathrm{~dB}-\mathrm{Hz}$.

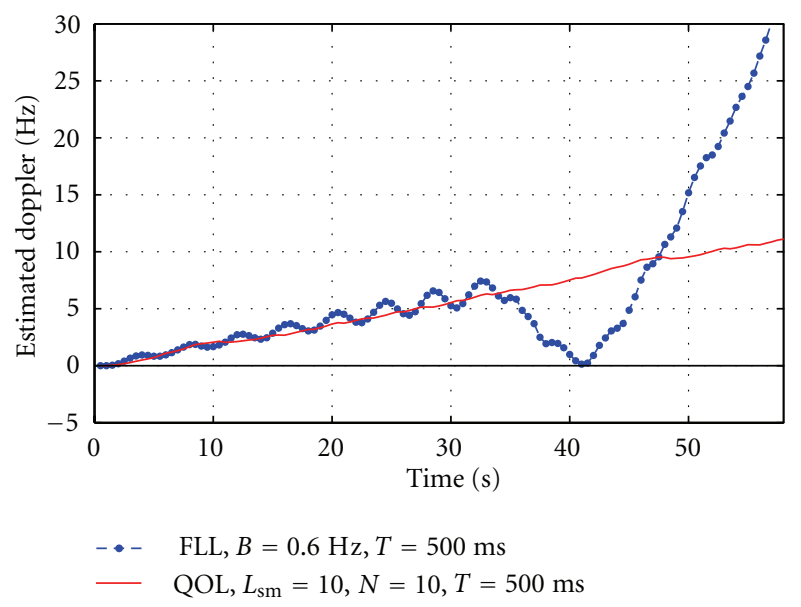

FIGURe 15: FLL versus QOL tracking results for incoming Doppler variation of $0.2 \mathrm{~Hz} / \mathrm{s}, C / N_{o}=20 \mathrm{~dB}-\mathrm{Hz}$. 
with a time-variant length. The number of the taps is set to one at each new updating, and then it is gradually increased to reach the specified maximum value $L_{\mathrm{sm}}$. The results are presented in Figure 10, where we can see that we have no more jumps in the output.

At this point we have an architecture where it is possible to set the update rate of the NCO independently from the integration time. We have also demonstrated the feasibility of using an MA-FIR filter with a time-varying length as loop filter. The filter design is very easy and the resulting structure is always stable.

\subsection{Performance of the Quasi-Open Scheme in User Dynamics.} The updating interval in the proposed scheme is strictly related to the dynamics under which the system has to work. In case of high dynamic applications the updating has to be applied more frequently, and this implies a smaller value of $N$, and vice versa. The dynamic range sustained by the quasi-open loop scheme does not only depend on $N$, but also on the linear region of the discriminator in (14), which, in turn, depends on the integration time $T$. We can relate the linear range of the discriminator, denoted by $\Delta_{D}$ (for a given $T$ ), and the rate of change of the input Doppler frequency, denoted by $\dot{f}_{d}$ and expressed in $\mathrm{Hz} / \mathrm{s}$ as

$$
\dot{f}_{d} T N<\frac{\Delta_{D}}{2}
$$

obtaining the condition for the quasi-open loop scheme to work properly. In this equation, the term $\dot{f}_{d} T N$ indicates the amount of Doppler accumulated between two updating intervals of the NCO. If this term lies inside the discriminator range, then the proposed scheme successfully tracks the Doppler variations.

As an example, for $T=10 \mathrm{~ms}$, the linear range of the discriminator is about $\Delta_{D}=100 \mathrm{~Hz}$ (from $-50 \mathrm{~Hz}$ to $50 \mathrm{~Hz}$ in Figure 5). Thus, for $T=10 \mathrm{~ms}$ and $N=20$, the maximum Doppler variation that can be tracked successfully by the quasi-open loop scheme is given by

$$
\dot{f}_{d}<\frac{\Delta_{D}}{2 N T}=250 \mathrm{~Hz} / \mathrm{s}
$$

If the input Doppler variation exceeds this value, then the quasi-open loop is not able to track the incoming frequency. The problem can be solved by updating the NCO frequency more often. This can be achieved by decreasing the value of N.

\subsection{Tracking Jitter Performance. We carried out some sim-} ulation experiments to examine the tracking jitter performance of the proposed quasi-open loop scheme and we compared it with the theoretical jitter of a classical FLL. The results shown in Figure 11 were obtained for different integration times $T$ and different $C / N_{o}$, by keeping the number $L_{\mathrm{sm}}$ of the MA-FIR filter taps constant, and by updating the NCO (local oscillator $\mathrm{LO}(\mathrm{E})$ ) frequency after $N=20$ epochs. In this way we were able to analyze the effect of increasing the integration time on the jitter performance.
To compare the results with those of a traditional closed loop scheme, we used the formula of the theoretical FLL tracking jitter, due to thermal noise, given in [2], that is

$$
\sigma_{\mathrm{FLL}}=\frac{1}{2 \pi T} \sqrt{\frac{4 F B}{C / N_{o}}\left[1+\frac{1}{T C / N_{o}}\right]}[\mathrm{Hz}] .
$$

These values are also shown in Figure 11 for different integration times, and for a loop noise bandwidth $B=$ $10 \mathrm{~Hz}$. The choice of the loop noise bandwidth suitable for comparison is not an easy task and will be discussed in the next section.

It is clear from the obtained results that the tracking jitter performance of the quasi-open loop is better than that of an FLL for all the integration times at high $C / N_{o}$, but the performance degrades at low $C / N_{o}$. The main reason for this could be the poor quality of the frequency estimator (14) at a low $C / N_{o}$.

5.4. Loop Noise Bandwidth versus Filter Taps. In this section, we discuss the equivalence between the loop noise bandwidth $B$ of the closed loop schemes and the number $L_{\text {sm }}$ of the filter taps in a quasi-open loop. In traditional closed loop schemes, like FLLs, the parameter $B$ controls both the amount of noise rejected by the loop and the dynamic stress. If we increase $B$, the loop can sustain more dynamics, but more noise also affects the system, and vice versa.

A similar role is played by the number $L_{\mathrm{sm}}$ of filter taps in a quasi-open scheme. Increasing $L_{\mathrm{sm}}$ implies more smoothing, but imposes some constraints on the update interval $N$, which should be larger than $L_{\text {sm }}$. In fact, if $N<$ $L_{\text {sm }}$ the filter never attains steady-state, thereby reducing the effectiveness of the smoothing, which will be incomplete. In other words, since the number of taps is variable and is set to one at each new update, the maximum number of taps will be $N$ instead of $L_{\mathrm{sm}}$. Another constraint on the value of $N$ is the dynamic stress which can be sustained by the quasi-open loop scheme.

The equivalence between $B$ and $L_{\mathrm{sm}}$ can be seen in Figure 12, which shows the tracking jitter curves for both schemes, with different values of $B$ for the FLL and different values of $L_{\mathrm{sm}}$ for the quasi-open scheme. The curves were obtained by keeping the integration time constant. By observing these curves we can get an idea of the number of taps of the MA-FIR filter required to achieve the same jitter performance of an FLL with a given noise bandwidth. As shown in Figure 12, the jitter performance improves as $L_{\mathrm{sm}}$ increases, and $B$ decreases.

It is very difficult to find the exact equivalence between these two parameters from these results. However, since we are usually interested in low $C / N_{o}$ regions, we can somehow relate the two parameters. For example, to obtain the same performance of an FLL with $B=10 \mathrm{~Hz}$, we need an MAFIR filter with $L_{\mathrm{sm}}=20$ taps for $T=1 \mathrm{~ms}$. On the other hand, for $B=1 \mathrm{~Hz}$, the number of taps has to be $L_{\mathrm{sm}}=50$, which is quite complex in terms of computational cost. So, a reasonable choice could be to fix an approximate limit of $L_{\mathrm{sm}} \leq 20$. In this way we will get almost the same performance of an FLL with $B=10 \mathrm{~Hz}$. 
In the following section we adopt this equivalence between $B$ and $L_{\mathrm{sm}}$, and, based on this, we show the advantages obtained with a quasi-open loop scheme in weak signal conditions.

5.5. Weak Signal Performance. In this section, we demonstrate that, by using a quasi-open scheme, we can work with extended coherent integration times which otherwise would not be possible with closed loop schemes under the same conditions, because of the resulting loop filter instability.

In the first simulation experiment, we again considered an incoming signal with a ramp-type Doppler frequency with a slope of $10 \mathrm{~Hz} / \mathrm{s}$, and with $C / N_{o}=40 \mathrm{~dB}-\mathrm{Hz}$. Although this is not a weak signal, we started with this value to better highlight the performance of both schemes, FLL and quasiopen loop, when they work under the same conditions. We set the update interval to $N=20$, and the number of filter taps to $L_{\mathrm{sm}}=10$. For this choice of $L_{\mathrm{sm}}$, a reasonable value of the FLL noise bandwidth could be around $B=10 \mathrm{~Hz}$, as discussed in Section 5.4. The tracking results are shown in Figure 13 for $T=30 \mathrm{~ms}$. Under these conditions $B T=$ 0.3 , which is high enough for the loop filter to become unstable. For the quasi-open loop scheme we can extend the integration time even more as long as the condition (16) remains valid.

In the next simulation experiment, we considered a weak signal with $C / N_{o}=30 \mathrm{~dB}-\mathrm{Hz}$, and with a Doppler varying at a rate of $0.5 \mathrm{~Hz} / \mathrm{s}$. Since $C / N_{o}$ is very low, we set $T=300 \mathrm{~ms}$, and the FLL bandwidth to $B=1 \mathrm{~Hz}$, so as to have a suitable noise rejection performance for weak signals. For the quasiopen loop scheme, we chose $L_{\mathrm{sm}}=10$ and $N=10$ to fulfill the condition (16). Here the value $N=20$ used in the previous experiment cannot be kept because of condition (16). The results are shown in Figure 14. Again we observe the same situation observed in Figure 13: the FLL is no longer able to track the carrier, because of the loop filter instability, while the proposed scheme works well and successfully tracks the incoming Doppler variation.

Figure 15 shows the tracking results for an incoming signal with $C / N_{o}=20 \mathrm{~dB}-\mathrm{Hz}$, and a Doppler variation with a rate of $0.2 \mathrm{~Hz} / \mathrm{s}$. The integration time is set to $T=500 \mathrm{~ms}$, since $C / N_{o}$ is very low. For the quasi-open loop scheme, we chose $N=10$, a value which does not violate the condition (16). The figure shows that the proposed scheme successfully tracks the frequency of the incoming signal even at this low $\mathrm{C} / \mathrm{N}_{\mathrm{o}}$.

\section{Conclusion}

A novel quasi-open loop architecture has been proposed for tracking the frequency of received GNSS signals. The proposed architecture works with three different rates, unlike the classical closed loop schemes, PLLs and FLLs, which work with two different rates. The additional degree of freedom of the quasi-open scheme enables us to ease the design of the loop filter. Simulation results show that it is possible to design this filter with an FIR structure, by adopting some very simple rules for the design. Moreover, the system results advantageous also in terms of stability when compared to a traditional closed loop architecture. It is also important to mention that if this type of scheme is used with classical DPLL schemes in an assisted manner, then a lower bandwidth and higher coherent integration times can be utilized in DPLL to track the incoming carrier in very weak signal conditions.

\section{References}

[1] B. Parkinson and J. Spilker, Eds., Global Positioning System: Theory and Applications, vol. 1, American Institute of Aeronautics and Astronautics, Washington, DC, USA, 1996.

[2] E. Kaplan and C. Hegarty, Eds., Understanding GPS Principles and Applications, Artech House, Norwood, Mass, USA, 2006.

[3] P. L. Kazemi, Development of new filter and tracking schemes for weak gps signal tracking, Ph.D. dissertation, Department of Geomatics Engineering, University of Calgary, 2010.

[4] S. A. Stephens and J. B. Thomas, "Controlled-root formulation for digital phase-locked loops," IEEE Transactions on Aerospace and Electronic Systems, vol. 31, no. 1, pp. 78-95, 1995.

[5] P. L. Kazemi, "Optimum digital filters for GNSS tracking loops," in Proceedings of the 21st International Technical Meeting of the Satellite Division of the Institute of Navigation (ION GNSS '08), pp. 1188-1197, Center Savannah, Ga, USA, September 2008.

[6] M. L. Psiaki, "Smoother-based GPS signal tracking in a software receiver," in Proceedings of the 14th International Technical Meeting of the Satellite Division of The Institute of Navigation (ION GPS '01), pp. 2900-2913, Salt Palace Convention Center, Salt Lake City, Utah, USA, 2001.

[7] A. M. M. Kamel, Context aware high dynamics GNSS-INS for interference mitigation, Ph.D. dissertation, Department of Geomatics Engineering, University of Calgary, 2011.

[8] J. Riba, J. Tom, and M. Lagunas, "Instantaneous openloop frequency estimation methods for navigation receivers," in Proceedings of the 2nd European Symposium on Global Navigation Satellite Systems (GNSS'98), Toulouse, France, 1998.

[9] S. Kay, "Fast and accurate single frequency estimator," IEEE Transactions on Acoustics, Speech, and Signal Processing, vol. 37, no. 12, pp. 1987-1990, 1989.

[10] F. van Graas, A. Soloviev, M. Uijt de Haag, and S. Gunawardena, "Closed-loop sequential signal processing and openloop batch processing approaches for GNSS receiver design," IEEE Journal on Selected Topics in Signal Processing, vol. 3, no. 4, pp. 571-586, 2009.

[11] M. Tahir, M. Fantino, and L. L. Presti, "Characterizing different open loop fine frequency estimation methods for GNSS receivers," in Proceedings of the International Technical Meeting of The Institute of Navigation (ION ITM '12), Newport Beach, Calif, USA, 2012.

[12] M. L. Fowler, "Phase-based frequency estimation: a review," Digital Signal Processing, vol. 12, no. 4, pp. 590-615, 2002.

[13] D. Simon and H. El-Sherief, "Fuzzy logic for digital phaselocked loop filter design," IEEE Transactions on Fuzzy Systems, vol. 3, no. 2, pp. 211-218, 1995. 

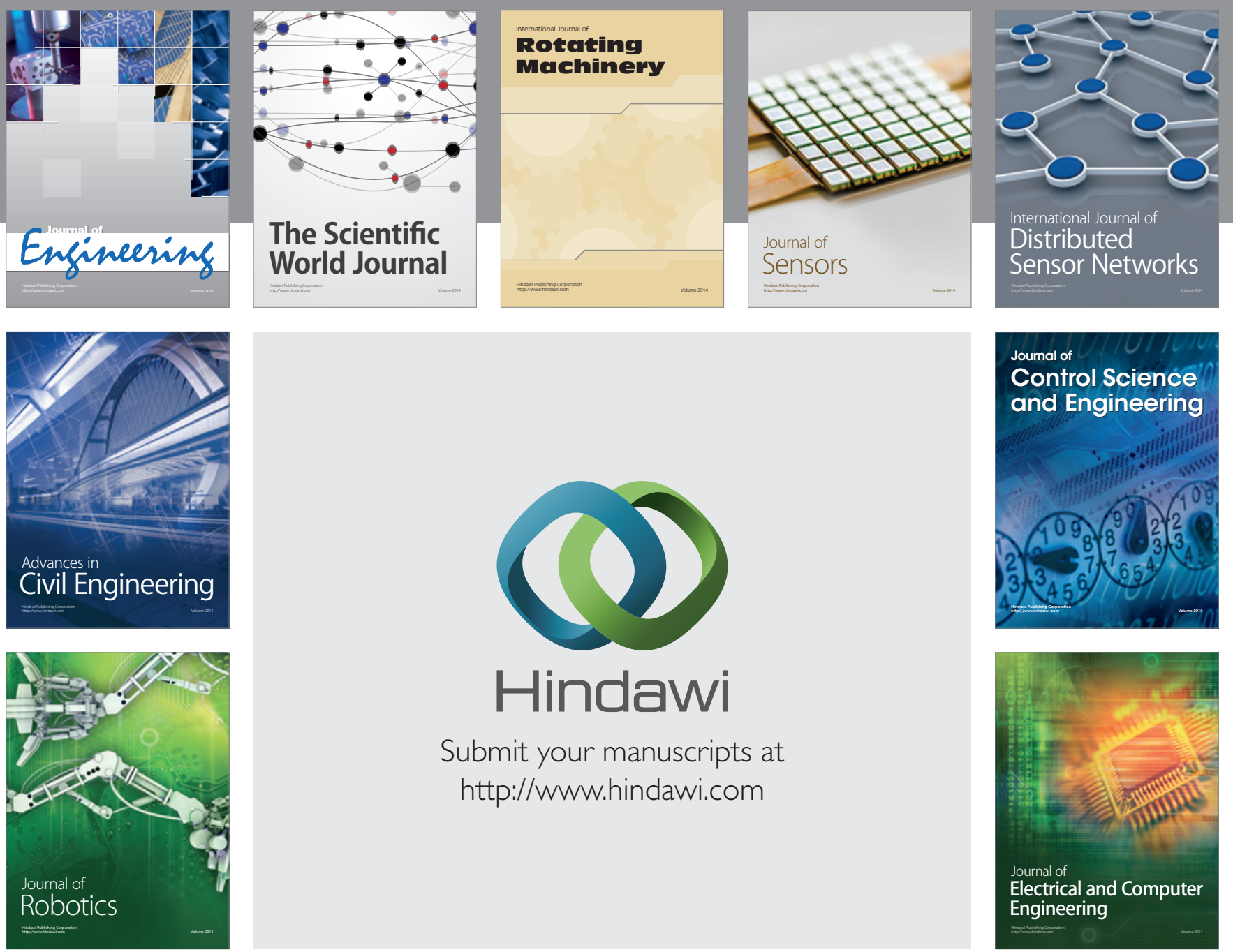

Submit your manuscripts at

http://www.hindawi.com
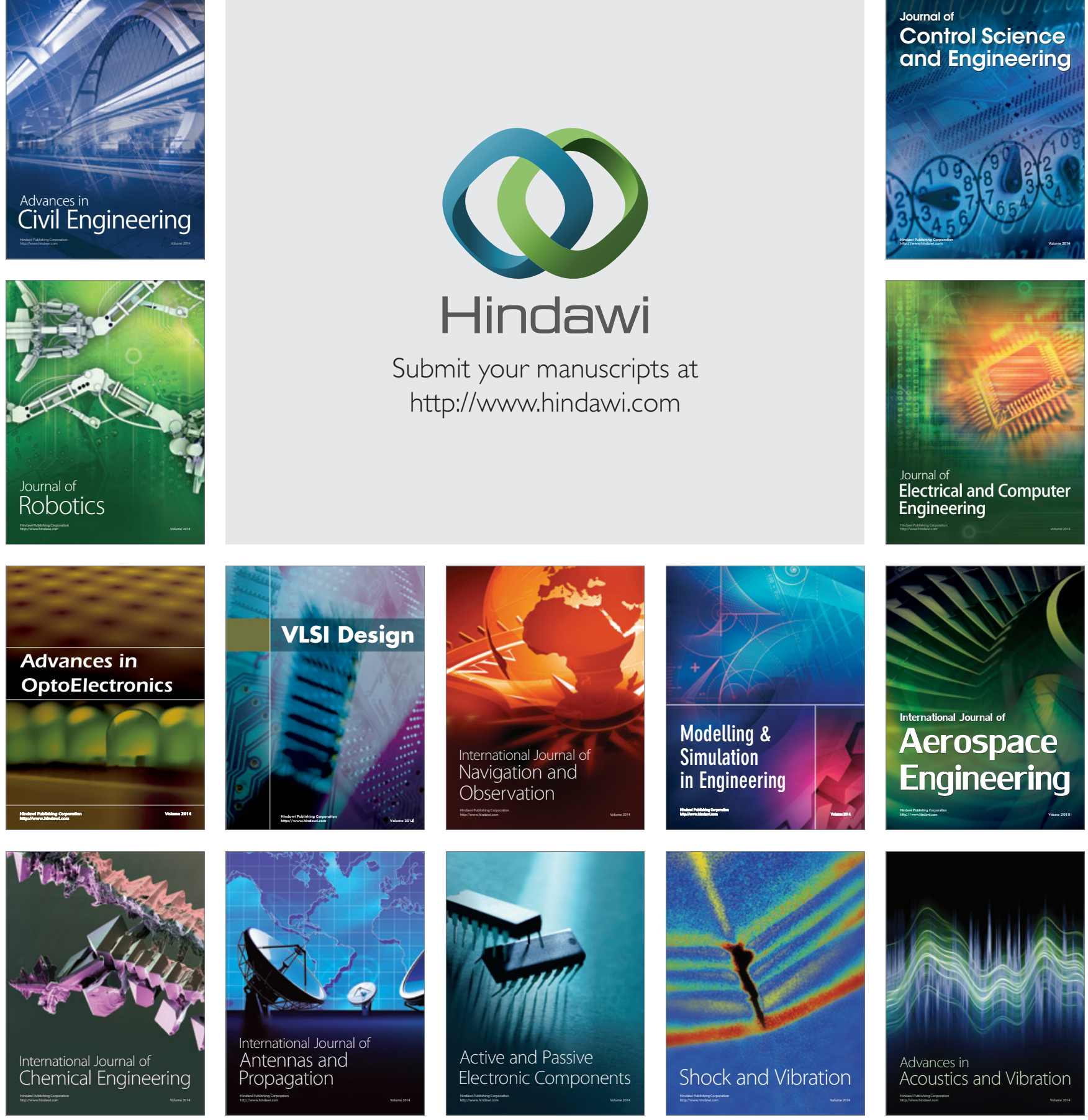